J. Phys. IV France 134 (2006) 473-479

(C) EDP Sciences, Les Ulis

DOI: $10.1051 /$ jp4:2006134073

\title{
Pulse mitigation by a composite discrete medium
}

\author{
C. Daraio1 ${ }^{1}$, V.F. Nesterenko ${ }^{1,2}$, E.B. Herbold ${ }^{2}$ and S. Jin ${ }^{1,2}$ \\ ${ }^{1}$ Materials Science and Engineering Program \\ 2 Department of Mechanical and Aerospace Engineering, University of California \\ at San Diego, La Jolla, CA 92093-0411, USA
}

\begin{abstract}
The strongly nonlinear interaction between elements in discrete materials (e.g., grains in granular media) is responsible for their unique wave propagation properties. The paper will present an experimental observation of impulse energy confinement and the resultant disintegration of shock and solitary waves by discrete materials with strongly nonlinear interaction between elements. Experiments and numerical calculations will be presented for alternating ensembles of high-modulus vs orders of magnitude lowermodulus chains of spheres of different masses. The trapped energy is contained within the "softer" portions of the composite chain and is slowly released in the form of weak, separated pulses over an extended period of time. This effect is enhanced by using a specific group assembly and a superimposed force.
\end{abstract}

\section{INTRODUCTION}

One-dimensional chains of beads represent the simplest example of strongly nonlinear systems. They exhibit a very unique dynamic behavior, especially at the interface between two different granular systems [1-5] or at the interface between the granular media and solid matter [6]. In the past, the design of shock protectors focused mainly on the enhanced energy dissipation provided by layered systems or by the friction in granular media, i.e. granular beds composed of iron shot [2] (the waste from metallurgical plants). Yet another way of protecting materials may be realized exploiting the unique dynamic properties observed at the interface of two strongly nonlinear media. Theoretical analysis $[3,4]$ predicted the possibility of designing a novel shielding medium called a "granular container" acting through the confinement of an impulse. The study of these one-dimensional cases is of general interest for developing the fundamental understanding of the strongly nonlinear behavior of these complex media. Practical applications can subsequently arise extending the properties of the 1-D system to a three dimensional array of ordered granular chains [7] facilitating the development of metamaterials with strongly nonlinear properties for various applications, as it has happened in the case of weakly nonlinear wave dynamics [8].

The nonlinear Hertzian contact interactions between the elastic spherical particles composing the system is responsible for the dynamic nonlinearity and results in a power-law type dependence of the compressive force $(F)$ on displacement $(\delta)$. In the case of zero or very weak precompression (resulting in zero or very small sound speed, i.e. "sonic vacuum" (SV) type systems) the corresponding wave equation supports a qualitatively new solitary wave [2]. A peculiar property of the granular media derives from the possibility of "tuning" the type of stationary solution produced by the system by varying the precompression acting on the chains $[2,9,10]$. This allows "choosing" the regime of wave propagation or the reflection from the interfaces of two SVs according to the needs for each specific application.

The passage of a solitary wave through the interface of two SV type systems from a region of particles with a higher elastic modulus (or higher mass) to a region of lower elastic modulus (or lower mass) results in the impulse decomposition into a train of solitary pulses $[1-5,11]$. The reflection from light and heavy inclusions was earlier proposed as a technique for nondestructive identification of impurities in a granular medium (with implications in the analysis/detection in geological or biological fields) [12]. 
In this paper we present some important steps in understanding the interface dynamics of composite 1-D strongly nonlinear systems based on polymer/steel chains under different configurations. Firstly we analyze the behavior of pulses propagating through a single interface, and secondly we present the design of an efficient energy trapping device as an active protector.

\section{EXPERIMENTS AND NUMERICAL ANALYSIS}

To study the properties of interface interaction we assembled chains of nonmagnetic stainless steel particles, PTFE (polytetrafluoroethylene) beads and Parylene-C coated steel beads in different configurations. Piezogauges were placed inside particles measuring the averaged compression forces between the corresponding two contacts as in [13]. Magnetically induced precompression was induced on the system adding a Neodymium-Iron-Boron ring magnet (internal and external diameters $11.43 \mathrm{~mm}$, $53.34 \mathrm{~mm}$ respectively, with mass $242.2 \mathrm{~g}$ from Dexter Magnetics Technologies) around the PTFE cylinder containing the chain. Throughout the whole chain the precompressive force so obtained was $2.38 \mathrm{~N}$. For comparison with experiments a system of second order differential equations was reduced to the first order equations and numerical calculations were performed using Matlab considering particles as rigid bodies connected by nonlinear springs according to Hertz law for contact interaction of spheres.
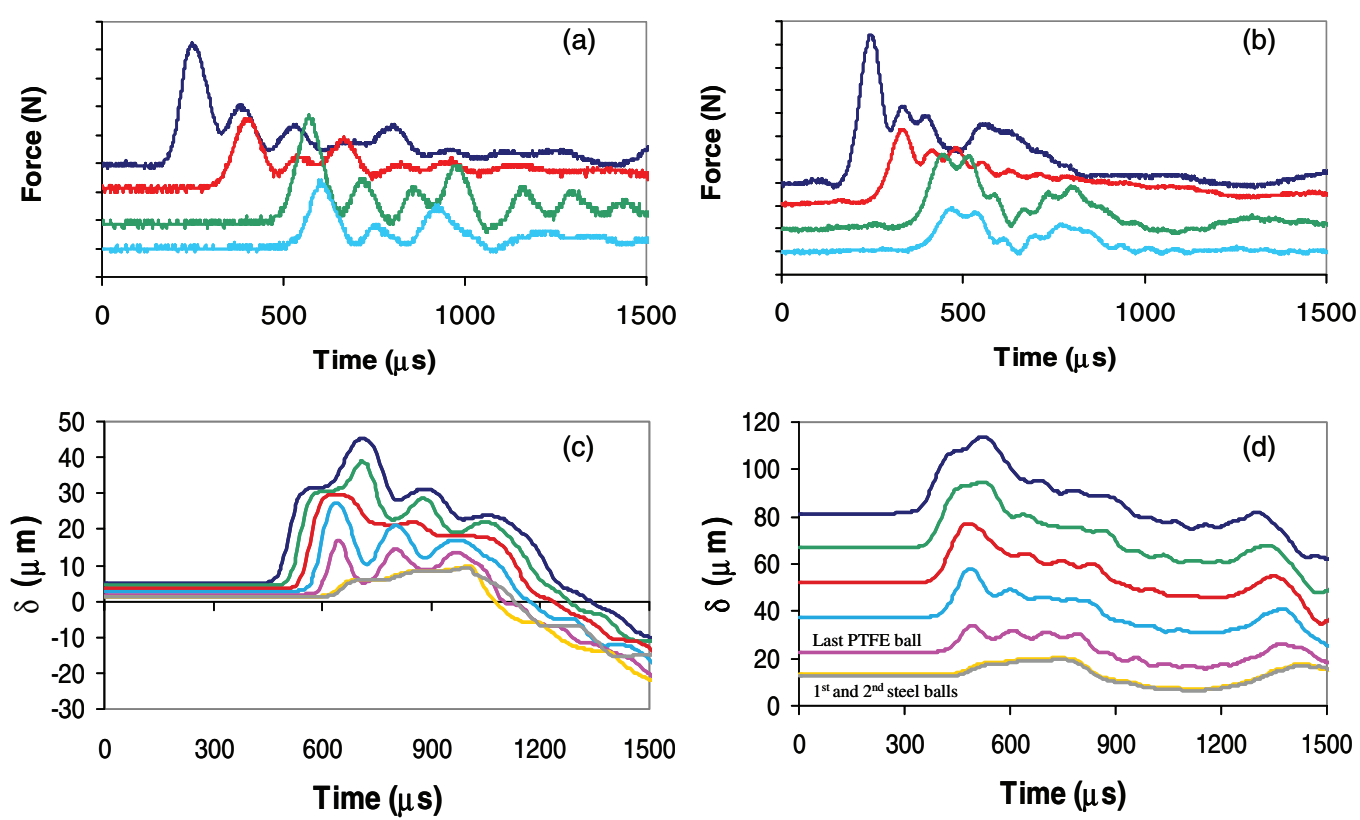

Figure 1. Pulse interaction with the interface of the two SVs composed of particles with different masses and elastic moduli with incident pulse coming from the "soft" section. The corresponding sections are composed from 20 PTFE and 21 stainless steel beads. An additional magnetic steel particle is added on the top of the PTFE chain to allow magnetic precompression. In (a) and (b) the curves corresponds to sensors placed in the 14th particle (8th particle from the interface), the 18th particle (4th from interface), the 25th particle (4th steel from the interface), and 28th particle (7th steel bead from the interface). (a) Experimental data with no static precompression. (b) Experimental data with added static precompression, vertical scales $0.2 \mathrm{~N}$ per division in (a) and (b). The $\mathrm{Al}_{2} \mathrm{O}_{3}$ striker $(0.47 \mathrm{~g})$ impact velocity is $0.44 \mathrm{~m} / \mathrm{s}$ in both cases. (c) Displacements of the PTFE and stainless steel beads adjacent to the interface of the two SVs under only gravitational precompression. (d) Same as (c) but under gravitational and magnetic precompression. 
In the experiment corresponding to Figure 1 the soft/lighter (PTFE beads, $0.123 \mathrm{~g}$ and $\mathrm{E}=1.46 \mathrm{GPa}$ [13]) portion of the chain was placed on top and the hard/heavier (316 steel beads, $0.45 \mathrm{~g}$ and $\mathrm{E}=193 \mathrm{GPa}[14]$ ) portion of the chain at the bottom. The data corresponding to the wave behavior the non-magnetically precompressed chain is presented in Fig. 1(a) and the one obtained from the statically prestressed chain is shown in Fig. 1(b). Figure 1(c) and (d) show the displacement $(\delta)$ vs. time $(t)$ curves relative to the motion of the last 5 PTFE particles before the interface and the first 2 stainless steel beads after the interface, when testing under only gravitational precompression and under gravitational plus magnetically induced precompression correspondingly.

A second set of experiments was performed placing the steel beads chain on the top and the PTFE chain of beads at the bottom (inverted setup as compared to the Fig. 1 case). The experiments and corresponding numerical analysis are presented in Fig. 2 for the only gravitationally loaded chain (Fig. 2(a) and (b)) and the gravitationally and magnetically pre-compressed system (Fig. 2(c) and (d)). Similar experiments and numerical analysis were performed on the same system using an interfacial particle with a flat bottom side as a contact between the steel and PTFE portions of the system.
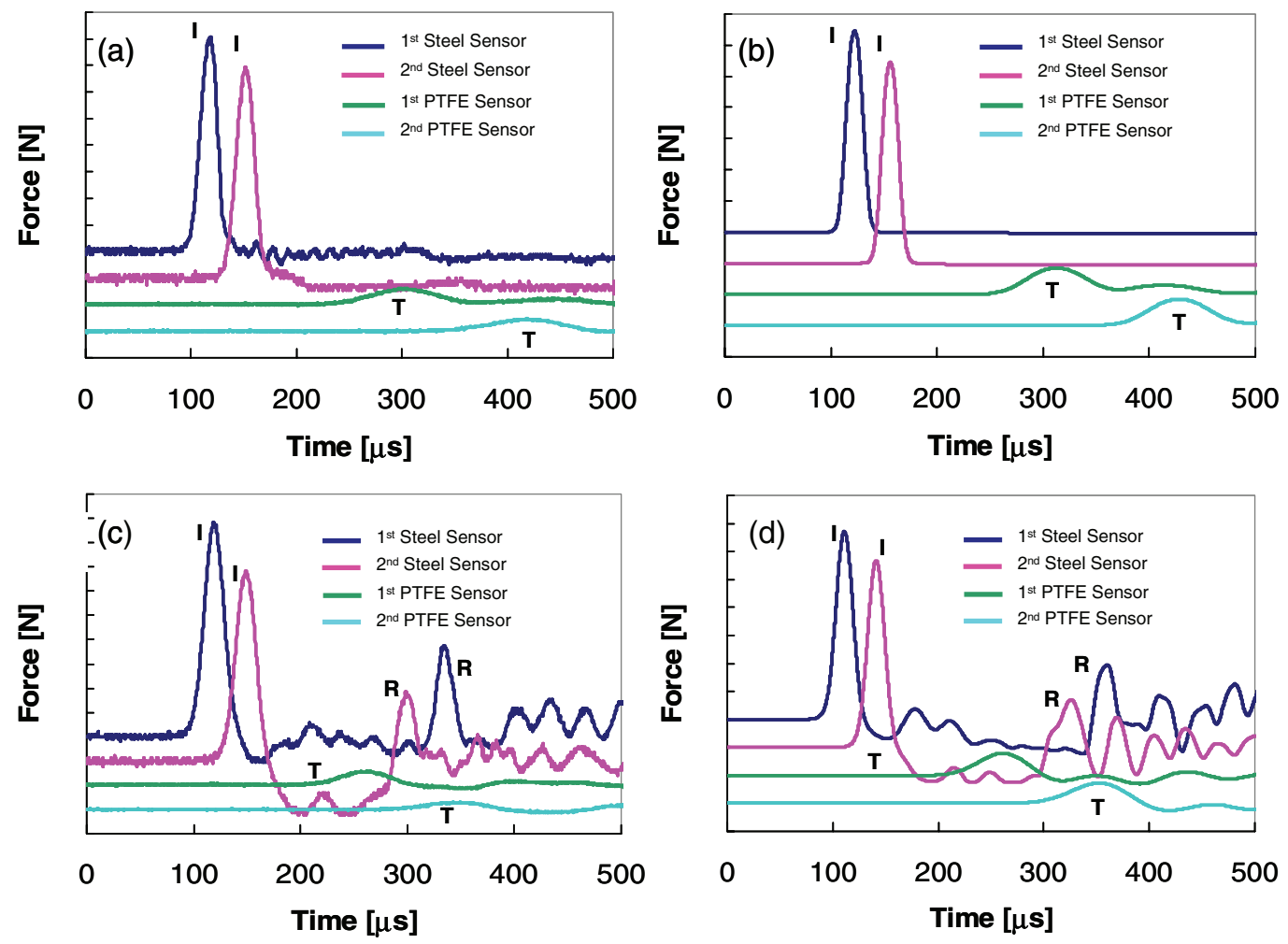

Figure 2. Pulse interaction with the interface of the two SVs composed of particles with different masses and elastic moduli with incident pulse coming from the "rigid" section [5]. The system is composed of 20 stainless steel particle on top and 21 PTFE beads at the bottom. An additional magnetic steel particle is added on the top of the stainless steel chain. In all figures sensors are placed in the 14th particle (8th particle from the interface), the 18th particle (4th from interface), the 25th particle (4th PTFE from the interface), and 29th particle (8th PTFE from the interface). (a) Experimental data with only gravitational preload, vertical scale $1 \mathrm{~N}$; (b) numerical simulation of (a), vertical scale $2 \mathrm{~N}$; (c) experimental data for gravitationally and magnetically (2.38 $\mathrm{N}$ ) precompressed chains, vertical scale $1 \mathrm{~N}$; (d) numerical simulation of $(\mathrm{c})$, vertical scale $2 \mathrm{~N}$. Impact was by alumina cylinder with mass $0.47 \mathrm{~g}$, velocity $0.44 \mathrm{~m} / \mathrm{s}$. 

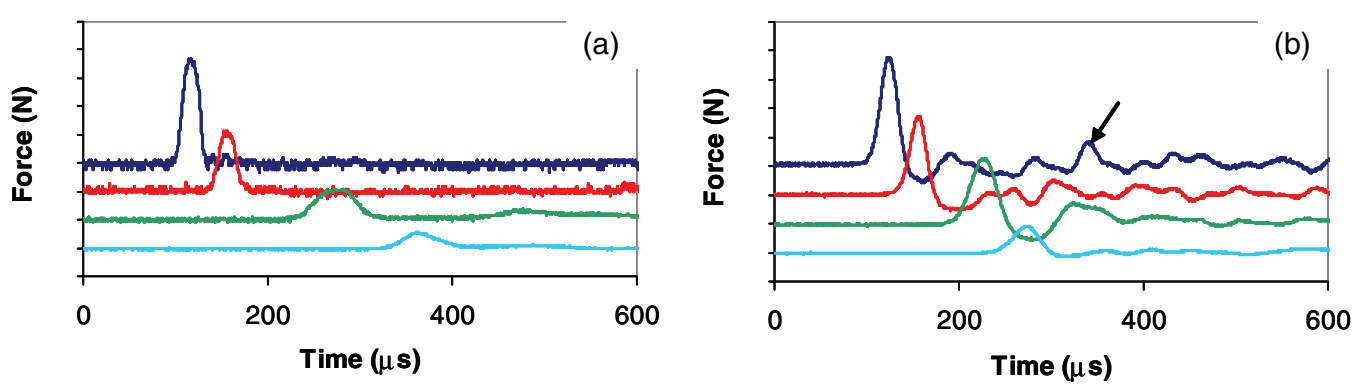

Figure 3. Pulse interaction with the interface of two SVs with similar masses of the particles with large difference in elastic moduli with incident pulse coming from "rigid" section. The system is composed of 20 stainless steel particle on top and 21 Parylene-C coated beads at the bottom. An additional magnetic steel particle is added on the top of the stainless steel chain. In all figures sensors are placed in the 14th particle (8th particle from the interface), the 18th particle (4th from interface), the 25th particle (4th Parylene-C coated from the interface), and 29th particle (8th Parylene-C coated from the interface). (a) Experimental data with only gravitational preload; (b) experimental data for gravitationally and magnetically $(2.38 \mathrm{~N})$ precompressed chains. The vertical scale of the first two curves is $2 \mathrm{~N}$ per division and for the bottom two $0.4 \mathrm{~N}$ Impact was by alumina cylinder with mass $0.47 \mathrm{~g}$, velocity $0.77 \mathrm{~m} / \mathrm{s}$. The arrow shows the presence of the anomalous reflected wave as observed in Fig. 2(c).

The flat interface was introduced to assure a Hertzian type of interaction between the very soft PTFE sphere and the rigid plane surface (stainless steel) in experiments and to monitor the wave propagation behavior when interfacial properties of the system have changed. An additional set of experiment to understand the phenomena of wave propagation behavior through a hard/soft interface was performed placing the chain of steel beads on the top of a chain composed of Parylene- $\mathrm{C}$ coated beads (see Figure 3). The peculiarity of this interface stems from the fact that the steel beads and the parylene coated beads have practically the same mass $(0.45 \mathrm{~g})$ but very different elastic modulus $(2.76 \mathrm{GPa}$ for the Parylene-C coated beads) [15].

A final set of experiments was performed on a composite system assembled from alternating ensembles of steel/PFTE beads in order to exploit the interfacial phenomena described earlier. In these experiments the beads were assembled as in Fig. 4(a) and the system was tested under single solitary pulse propagation and shock-like impacts (Fig. 4(b-d)), with and without the addition of static precompression.

\section{DISCUSSION}

The wave propagation at the interface of PTFE/Steel chains is described in Figure 1. Under the investigated conditions of impact a train of 4 solitary waves is quickly emerging at the vicinity of the impacted end (the mass of impactor and the mass of the end magnetic particle were about 4 times the mass of the beads in the chain). This is a typical feature of strongly nonlinear systems [2]. The interaction of the incident pulse with the interface generates both transmitted and reflected waves detected by the gauges in the PTFE and the steel chains. A good agreement between experimental data (Fig. 1(a)) and numerical analysis (not shown) is observed. Magnetically induced preloading significantly increased the speed of solitary waves [14] in PTFE and steel chains. A less pronounced splitting of the initial pulse into the train of solitary waves is evident both in experimental and numerical data although the character of the reflection was qualitatively the same. A reasonable agreement is observed between the experimental and numerical data.

When testing under similar conditions the "inversed" setup, placing the steel portion of the chain on the top without the addition of the magnetically induced static prestress (See Fig. 2(a) and (b)), we observed a practically complete energy transfer of the pulse into the PTFE chain and the absence of any reflected compression wave [5]. When the additional static prestress $(2.38 \mathrm{~N})$ is added 


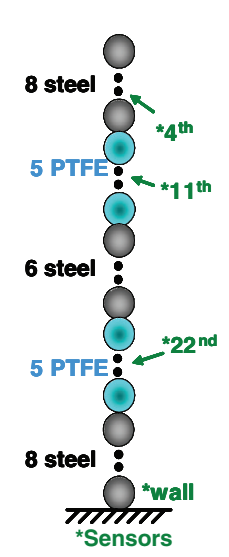

(a)
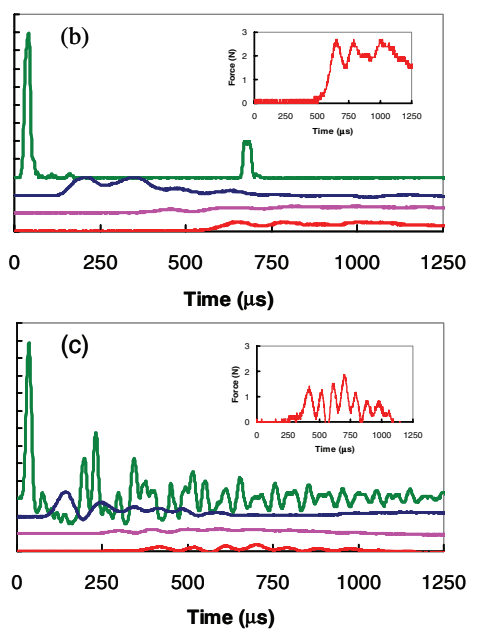
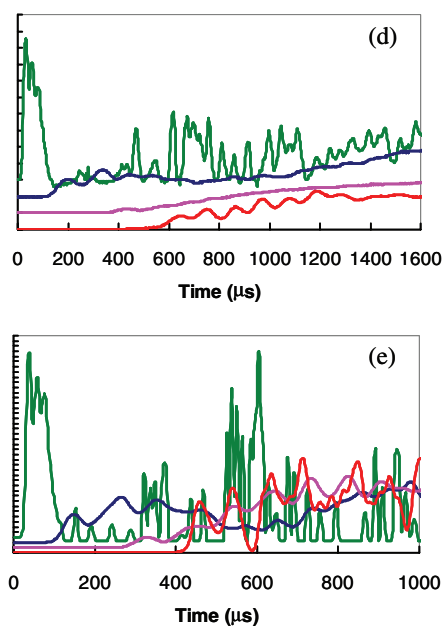

Figure 4. Solitary and shock-like pulse trapping in the composite double "granular container" with and without additional precompresion. (a) Schematic diagram of the arrangement of the stainless steel and PTFE beads with indicated sensors. (b) Experimental results for only gravitationally loaded system. (c) Experimental results with magnetically induced superimposed force, all other conditions as in (b). The y-axes scale is $1 \mathrm{~N}$ per division. Insets in $(b, c)$ show the pulse behavior at the wall for the gravitationally loaded and the magnetically tuned system. Note the significantly moderated impulse shape arriving on the wall in (b) and (c): the strong incident impulse (first curve) disintegrates into a very weak series of pulses delivered over a much longer period of time (bottom curve). (d) Shock pulse trapping obtained experimentally by the impact of an $\mathrm{Al}_{2} \mathrm{O}_{3}$ rod (63 g) striking with a velocity of $\sim 0.17 \mathrm{~m} / \mathrm{s}$. The y-axes scale is $1 \mathrm{~N}$. (e) Numerical analysis corresponding to (d).

(see Fig. 2(c) and (d) for experiments and numerical data correspondingly) an anomalous reflected wave was observed [5]. In addition to the expected reflected rarefaction wave we observed both in experiments and numerical calculations reflected compression waves! The leading reflected pulse has an amplitude of about one half of the amplitude of the incident wave. At the same time the leading transmitted compression pulse in PTFE chain is followed by unexpected rarefaction pulses. A peculiar characteristic of this reflected compression wave is related to its delayed time of arrival at the gauges inside stainless steel particles due to the opening and closing of gaps around the interface. This phenomenon mainly exists when the pulse is approaching the interface from the "stiffer" part of the system (larger elastic modulus, like in the case of the stainless steel/Parylene-C interface, or larger elastic modulus combined with larger mass as in the case of the stainless steel/PTFE interface). This difference is illustrated when compare Figs. 1(c) and (d) and Fig. 1(c) and 2(c) in [5].

The replacement of the last bead of the stainless steel chain with a bead of equal mass but flat bottom interface resulted in similar results under the same testing conditions. Only small wave propagation variations seem to appear when replacing the spherical steel particle at the interface with a flat steel interface, showing consistency of the anomalous reflection results even with a planar interface.

The testing of a system composed of steel and parylene-C coated steel beads arranged in a setup analogous to Fig. 2 showed that the variation of the elastic modulus of the beads above and below the interface is sufficient to generate the anomalous reflected pulse. Figure 3(b) shows the effects of precompression on the single solitary pulse propagation through such system. The black arrow points at the anomalous reflected wave detected by the sensors in the stainless steel portion of the chain, after the interaction with the Parylene-C bottom section.

Finally, we present a new and efficient pulse-disintegration structure (see Fig. 4(a)) that exploits the interface properties described in the earlier experiments. The pulse confinement in the softer region of the chain is related to the self assembly of gaps at the interfaces combined with the reflection of the pulse from the interfaces of the soft and rigid regions. These gaps allow the two softer regions of the chain to 
keep the energy trapped longer, therefore enhancing the protection of the wall. The gaps delay the wave reflection and propagation, and enhance the backward reflections from the heavy/light interfaces [11].

The efficiency of soliton-like pulse trapping is presented in Fig. 4 (b) and (c) in the composite system with and without the addition of pre-compressive forces accordingly [11]. Under shock-type loading a drastic modification of the signal ramp time at the wall was obtained (see Fig. 4 (d) and (e) for gravitationally and magnetically and gravitationally prestressed systems correspondingly). The introduction of a magnetically induced precompression divided the signal reaching the wall into a series of subdivided pulses reducing the total force impulse even further. If properly configured, these grouped composite media can be building blocks for powerful energy absorbers against impacts, and can be useful as efficient protectors for technological and security applications.

\section{CONCLUSIONS}

The behavior of strongly nonlinear solitary waves propagating at the interface of two strongly nonlinear media is described under different experimental conditions and with systems composed of different materials, varying the mass and elastic properties of the beads in the arrangements. The discovery of an anomalous reflected pulse generated under the presence of a magnetically induced precompression is presented and the results are then applied to design a novel energy trapping protective system. The effectiveness of the novel trapping medium is tested under solitary-like pulse loading and under sock-like loading. The system proved effective in both cases and opens an all new area of possible investigations and applications.

\section{Acknowledgments}

The authors wish to thank the National Science Foundation for the support of this work with Grant No. NSFDCMS03013220.

\section{References}

[1] Nesterenko V.F., Lazaridi A.N. and Sibiryakov E.B. "The decay of soliton at the contact of two Acoustic Vacuums" Prikl. Mekh. Tekh. Fiz. 2 (1995) 19 [J. Appl. Mech. Tech. Phys. 36 (1995) 166].

[2] Nesterenko V.F. Dynamics of Heterogeneous Materials (Springer-Verlag, NY, 2001) Chapter 1.

[3] Hong J. and $\mathrm{Xu}$ A. "Nondestructive identification of impurities in granular medium" Appl. Phys. Lett. 81 (2002) 4868.

[4] Hong J. "Universal Power-Law Decay of the Impulse Energy in Granular Protectors" Phys. Rev. Lett. 94 (2005) 108001.

[5] Nesterenko V.F., Daraio C., Herbold E.B. and Jin S. "Anomalous wave reflection at the interface of two strongly nonlinear granular media” Phys. Rev. Lett. 95 (2005) 158702.

[6] Job S., Melo F., Sen S. and Sokolow A. "How Hertzian Solitary Waves Interact with Boundaries in a 1D Granular Medium” Phys. Rev. Lett. 94 (2005) 178002.

[7] Daraio C., Nesterenko V. F. and Jin S. "Strongly nonlinear waves in 3D phononic crystals" In: Shock Compression of Condensed Matter-2003, edited by M.D. Furnish, Y.M. Gupta, and J.W. Forbes, (American Institute of Physics, AIP Conference Proceedings 706, 2004) pp. 197-201.

[8] Remoissenet M. Waves Called Solitons (Concepts and Experiments), (3rd revised and enlarged edition. Springer-Verlag, Berlin, 1999).

[9] Daraio, C., Nesterenko, V.F., Herbold, E. and Jin, S. "Tunability of solitary wave properties in one dimensional strongly nonlinear phononic crystals" Phys. Rev. E 73 (2006) 026610.

[10] Coste C., Falcon E. and Fauve S. "Solitary waves in a chain of beads under Hertz contact." Phys. Rev. E 56 (1997) 6104. 
[11] Daraio C., Nesterenko V.F., Herbold E.B. and Jin S. "Energy Trapping and Shock Disintegration in a Composite Granular Medium” Phys. Rev. Lett. 96 (2006) 058002 (see also http://arxiv.org/abs/cond-mat/0509622).

[12] Sen S., Manciu M. and Wright J.D. "Solitonlike pulses in perturbed and driven Hertzian chains and their possible applications in detecting buried impurities.” Phys. Rev. E 57 (1998) 2386.

[13] Daraio C., Nesterenko V.F., Herbold E.B. and Jin S. "Strongly nonlinear waves in a chain of Teflon beads" Phys. Rev E 72 (2005) 016603.

[14] ASM Metals Reference Book, $2^{\text {nd }}$ Edition pp. 268; also ASM Metals Handbook, Properties and Selection of Metals, $8^{\text {th }}$ Edition 1 pp. 52 (also www.MatWeb.com).

[15] Daraio C. and Nesterenko V.F. "Strongly nonlinear waves in a chain of polymer coated steel beads". Phys. Rev. E 73 (2006) 026612. 